\title{
TRANSIÇÃO DO MÉTODO SCRUM PARA SCRUMBAN COMO APOIO AO TRABALHO VIRTUAL
}

Arthur Vieira de Lima (arthurvdlima@gmail.com) - Pontifícia Universidade Católica de Minas Gerais (PUC-Minas).

Ana Carolina dos Santos Paes (anacarolinads.paes@gmail.com) - Pontifícia Universidade Católica de Minas Gerais (PUC-Minas).

Matheus Luiz Pontelo de Souza (mslzposa@gmail.com) - Pontifícia Universidade Católica de Minas Gerais (PUC-Minas).

\section{RESUMO}

O trabalho virtual vem ganhando espaço no mercado principalmente em startups, nas quais recursos são escassos, e toda economia de tempo e dinheiro tende a ser preferida. Neste contexto, problemas oriundos dessa gestão remota enfrentados por um empreendimento digital são comuns e meios para tentar sanar estes devem ser adotados viabilizando a execução dos projetos. Logo, esse artigo possui o objetivo de diagnosticar os gargalos do processo de gestão do projeto da startup estudada, executado via home office desde seu nascimento, e analisar os resultados da implementação o método Scrumban. Também como objetivo, essa obra vai referenciar o case de sucesso de outra empresa, que adota o método citado sob circunstâncias parecidas de ambiente de trabalho e tipo de produto. Em seguida, serão descritos os pontos melhorados e os que ainda podem ser melhorados. Após as entrevistas serão discutidos os problemas em destaque: falha de comunicação, falta de conhecimento de métodos de gestão além do Scrum e falta de controle dos processos de gestão.

Palavras chave: startup; empreendimento digital; scrumban; home office; gestão ágil

\section{INTRODUÇÃO}

As startups tem ganhado a atenção de inúmeras áreas de atuação devido à crescente ascensão da tecnologia, inovação e às crescentes exigências dos consumidores modernos. Mediante a dificuldade de inovar e a restrição dos recursos como dinheiro e desenvolvimento técnico, é 
percebida uma necessidade de que a gestão do projeto, alocação destes recursos e tomadas de decisões sejam realizados de forma eficaz. Para isso, métodos com esses objetivos surgiram, e continuam se desenvolvendo, para auxiliar os empreendedores a escolher os melhores caminhos (SOUZA et al.,2017).

O presente estudo tem como base o empreendedorismo digital (ED), caracterizado por novos produtos e serviços baseados na internet, o que, diferentemente do empreendedorismo tecnológico (ET), não utilizam tecnologia física (GIONES; BREM, 2017). A criação de uma startup digital como um novo negócio dentro ou não de uma empresa já existente é o que define o ED (ZAHEER; BREYER; DUMAY, 2019). Shen, Lindsay e Xu (2018) definem o ED como "novos negócios e a transformação de negócios existentes por meio da criação de novas tecnologias digitais ou de novos usos de tecnologias digitais existentes". Após o entendimento do conceito de empreendedorismo digital foi assimilado que a internet é o principal aliado desse tipo de negócio: devido a sua infraestrutura de operação ser totalmente baseada em computação em nuvem (GIONES; BREM, 2017), o que viabiliza o trabalho em equipe à distância.

A pandemia da Covid 19 propiciou ênfase no trabalho à distância, e o que estava se tornando tendência para startups, se tornou foco. Os avanços tecnológicos permitem o trabalho remoto e a colaboração em equipes virtuais, e a convergência para o regime de trabalho virtual é consequência do rápido desenvolvimento das tecnologias de informação (ABARCA; PALOSSANCHEZ; RUS-ARIAS, 2020). Em sua pesquisa, Abarca, Palos-Sanchez e Rus-Arias (2020) afirmam que o estudo nesta área tem se tornado cada vez mais importante: o medo de novas pandemias e a economia de custos com transportes certamente promoverão o avanço das tecnologias que possibilitam o home office. Porém, novos problemas tendem a aparecer, como falta de conhecimento dos empreendedores sobre alternativas de estilos de gestão, falhas de comunicação e de controle de processos.

Nesta obra, serão retratadas as dificuldades de comunicação e gestão enfrentadas pela startup $\mathrm{P}$, junto as soluções adotadas pela empresa D contra essas adversidades. Os dois negócios foram os objetos de estudo da pesquisa. Para o embasamento teórico, foram abordados os conceitos e princípios de três frameworks do mundo tecnológico: Scrum, Kanban e Scrumban, expondo alternativas de estilos de gestão. O objetivo geral deste artigo é entender os problemas enfrentados pela startup $P$ e analisar se houve melhoria na entrega dos resultados após as mudanças no processo de gestão do projeto, considerando o ambiente de trabalho virtual de toda a equipe. A gestão da organização, que era composta pela metodologia Scrum, buscava 
melhorar a comunicação da equipe e uma forma mais eficiente de metrificar as prioridades de execução do projeto.

Segundo Ferrão e Canedo (2015), o Scrum aplicado exclusivamente em determinados contextos organizacionais não têm se mostrado efetivo no que se refere ao gerenciamento de tarefas, necessitando assim, combinar-se com outro modelo, como o método Kanban, para que as expectativas de agilidade sejam mantidas. A comunicação interna da startup P dependia exclusivamente das reuniões de review (revisão) e planning (planejamento) de cada sprint, já que os horários disponíveis de trabalho para a realização das reuniões diárias requeridas pela metodologia Scrum eram incompatíveis entre os integrantes. Neste trabalho, foram analisados os pontos fracos da gestão do projeto, que usava em sua maioria os conceitos do Scrum, e foi constatado que vários aspectos do modelo Kanban eram mais adequados para a realidade da equipe. Assim, buscou-se reunir alguns dos pontos essenciais dos três métodos para a evolução da gestão.

Por fim, este artigo exemplifica o caso da Empresa D que adota o modelo Scrumban de gestão e que obteve sucesso com sua aplicação. Devido a isso, a startup P implementou o modelo Scrumban e os resultados obtidos foram descritos neste trabalho. Logo, essa obra pode colaborar para empreendimentos digitais, no qual anseiam melhorar a gestão do desenvolvimento dos projetos ou ao menos conhecer outras possibilidades para futuras aplicações.

\section{REVISÃO TEÓRICA}

\subsection{Gestão Ágil: Scrum e Kanban}

Entre os vários métodos de gestão, existem os métodos ágeis (AMBLER, 2002), também chamados de métodos leves (FOWLER, 2001). Carvalho e Rabechini (2011) comenta que estes são adaptativos e flexíveis em relação aos tradicionais e além disso, eles são indicados para cenários em que existe constante mudança de requisitos e os resultados devem ser entregues em pequenos espaços de tempo comparado à gestão tradicional.

Geralmente, essas metodologias dividem o desenvolvimento em diversas interações. No caso do Scrum, estas são chamadas de sprints e realizam entregas ao final de cada uma das interações, de forma que o cliente receba uma versão que no momento agregue o maior valor ao seu negócio. Assim, as mudanças de requisitos podem ser acompanhadas pelos gestores em cada ciclo e existe uma retroalimentação por parte do cliente para a equipe de desenvolvimento, o que reduz o risco do projeto. Tais métodos foram influenciados pelas melhores práticas da 
indústria Japonesa, particularmente pelos princípios da manufatura enxuta implementados pelas companhias Honda e Toyota (CARVALHO; RABECHINI, 2011).

O Kanban abordado nesta obra se deu com a evolução do "antigo" método Kanban da automotiva Toyota, com a inovadora gestão visual por cartões, que segundo Tarne (2011) “O Kanban foi integrado ao Sistema Toyota de Produção”. O termo japonês Kanban que deu origem ao método significa cartão de avisos. Esse framework ajudou os japoneses a minimizar a quantidade de estoque disponível, usando o princípio just-in-time para o fornecimento de peças. Era preciso haver uma maneira de sinalizar quando os funcionários estavam prontos para mais trabalho, e com a evolução do framework, estratégias para criar quadros ainda mais visuais apareceram.

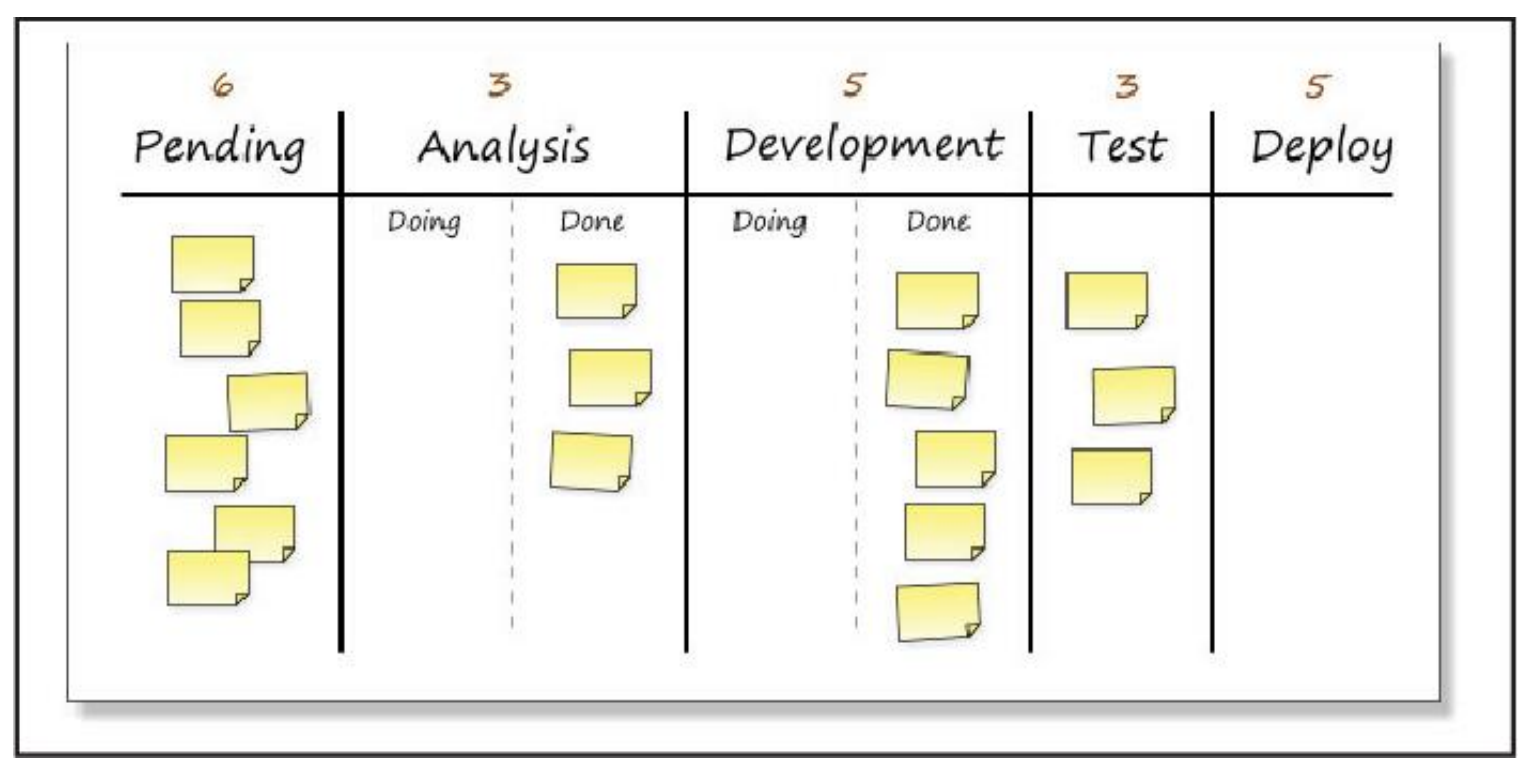

FIGURA 1 - Exemplo de Quadro Kanban. Fonte: Productivity Improvement of Software Development Process through Scrumban: A Practitioner's Approach (2018)

A obra de Alqudah e Razali (2018) explana que o fluxo de trabalho (work flow) é acompanhado e controlado de forma visual através quadro Kanban (Figura 1). "Isso restringe o trabalho em andamento via minimização do número de features (classes de tarefa mais detalhada) a serem implementados, medição e gerenciamento de vazão, a criação de políticas, implementação de rodadas consistentes de feedbacks e melhoria da colaboração" (ANDERSON, 2010). Comparando o método Kanban com o Scrum, o primeiro não detalha as práticas e princípios que exige, assim como o segundo tem diversas regras a serem seguidas. No entanto, devido à sua capacidade de lidar com os desafios que não poderiam ser enfrentados por métodos 
passados, o Kanban é considerado um método potente (SHALLOWAY, 2011). Além disso, o Kanban também mostra melhor eficácia para as equipes que executam o valor do negócio (ALQUDAH; RAZALI, 2018).

O método Scrum segue os princípios do Manifesto Ágil de 2001 e, segundo Schwaber e Beedle (2002), tem como objetivo definir um processo de desenvolvimento de projetos focado nas pessoas da equipe. De acordo com o estudo Alqudah e Razali (2018), baseado em sua maioria no 'Guia do Scrum' escrito por Sutherland e Schwaber, o método Scrum refere-se a uma abordagem que permite a geração e manutenção de produtos multifacetados. É simples e leve, mas a compreensão e implementação ainda são um desafio. No entanto, o Scrum permite o uso de diferentes processos e métodos entre os membros da equipe (SUTHERLAND; SCHWABER, 2013). Ele adere às regras que vinculam os eventos, funções e artefatos enquanto também controla os relacionamentos e interações que ocorrem entre eles. Aqui, a ênfase está na organização de equipes não motivadas por membros externos (ALQUDAH; RAZALI, 2018).

A equipe scrum é composta pelo Product Owner (PO), Scrum Master (SM) e uma equipe de desenvolvimento (SUTHERLAND; SCHWABER, 2013) que se interagem principalmente através de sprints, que são reuniões cíclicas para rever as entregas e promover as novas tarefas a serem executadas no próximo ciclo (geralmente o ciclo dura entre 1 e 3 semanas). No Scrum, cada equipe realiza suas tarefas de acordo com quatro artefatos: Backlog do Produto, Sprint Backlog, Incremento do Produto e definição concluída. Também, existem cinco tarefas a serem realizadas pela equipe Scrum para atingir os objetivos: Refinamento do Backlog, Reunião diária (chamada de daily scrum ou stand-up meeting), Planejamento (sprint planning), Retrospectiva (sprint retro) e Revisões, considerando cada uma das sprints. Desta forma, observa-se que o método Scrum é visto como um processo prescritivo (ALQUDAH; RAZALI, 2018).

Entretanto, segundo Ferrão e Canedo (2015), o Scrum aplicado exclusivamente em determinados contextos organizacionais, não têm se mostrado efetivo no que se refere ao gerenciamento de tarefas, necessitando assim de combinar-se com outro modelo, como o método Kanban, para que as expectativas de agilidade sejam mantidas. Além disso, o Scrum possui papéis essenciais ao seu funcionamento. O Kanban, no entanto, não prescreve qualquer tipo de papel em sua aplicação. Isso não significa que não se possa ter o papel de um $P O$ em Kanban. Porém, o excesso de papéis pode tornar-se um problema no contexto organizacional do processo, por isso utilizar somente o que é considerado necessário e essencial ao negócio, pode ser uma melhor abordagem (KNIBERG; SKARIN, 2010). 


\subsection{ScrumBan: Junção e Comparação}

O ScrumBan é um método ágil híbrido de Scrum e Kanban. Em busca de um método otimizado se faz essa mistura, a fim de utilizar os melhores pontos de cada um dos dois métodos. Portanto, é necessária uma hibridação de Scrum e Kanban para aprimorar o método Scrum, omitindo suas práticas inadequadas e adotando práticas apropriadas do método Kanban (ALQUDAH; RAZALI, 2018). A Figura 2 apresenta uma visualização do contexto híbrido comentado e foi usado para embasar o estudo de caso da startup P e da empresa D, ambos objetos de pesquisa do presente trabalho.

\begin{tabular}{|c|c|c|}
\hline Kanban & Scrum & Scrumban \\
\hline $\begin{array}{c}\text { Não há membros predefinidos } \\
\text { para funções }\end{array}$ & $\begin{array}{c}\text { Funções predefinidas do } \\
\text { Scrummaster e membros da } \\
\text { equipe }\end{array}$ & $\begin{array}{l}\text { Funções predefinidas do } \\
\text { Scrummaster e membros da } \\
\text { equipe podem variar dentro do } \\
\text { tempo do projeto }\end{array}$ \\
\hline Entrega contínua & Sprints de tempo fixo & $\begin{array}{c}\text { Iterações baseadas em quadro } \\
\text { de tarefas }\end{array}$ \\
\hline $\begin{array}{l}\text { WIP limita a quantidade de } \\
\text { trabalho }\end{array}$ & $\begin{array}{l}\text { Sprint limita a quantidade de } \\
\text { trabalho }\end{array}$ & $\begin{array}{l}\text { WIP limita a quantidade de } \\
\text { trabalho }\end{array}$ \\
\hline $\begin{array}{l}\text { Alterações podem ser feitas a } \\
\text { qualquer momento }\end{array}$ & $\begin{array}{c}\text { Nenhuma alteração permitida } \\
\text { durante os sprints }\end{array}$ & $\begin{array}{l}\text { Alterações permitidas no meio } \\
\text { do sprint }\end{array}$ \\
\hline $\begin{array}{l}\text { Planejamento e documentação } \\
\text { anteriores necessários }\end{array}$ & $\begin{array}{c}\text { Planejamento feito após cada } \\
\text { sprint }\end{array}$ & $\begin{array}{l}\text { Planejamento sob demanda, } \\
\text { também dentro de sprints }\end{array}$ \\
\hline $\begin{array}{l}\text { O quadro Kanban é } \\
\text { persistente }\end{array}$ & $\begin{array}{l}\text { O quadro scrum é redefinido } \\
\text { após cada sprint }\end{array}$ & $\begin{array}{l}\text { O quadro Scrumban é } \\
\text { persistente }\end{array}$ \\
\hline $\begin{array}{l}\text { O tamanho da tarefa não é } \\
\text { limitado }\end{array}$ & $\begin{array}{c}\text { Tamanho da tarefa limitada a } \\
\text { uma impressão }\end{array}$ & $\begin{array}{c}\text { O tamanho da tarefa não é } \\
\text { limitado }\end{array}$ \\
\hline $\begin{array}{l}\text { Gerenciamento de trabalho } \\
\text { baseado em puxar }\end{array}$ & $\begin{array}{l}\text { Gerenciamento de trabalho } \\
\text { baseado em backlog da Sprint }\end{array}$ & $\begin{array}{l}\text { Gerenciamento de trabalho } \\
\text { baseado em puxar }\end{array}$ \\
\hline
\end{tabular}

FIGURA 2 - Semelhanças e diferenças entre Scrum, Kanban e Scrumban. Fonte: Adaptado de Empirical Investigation of Scrumban in Global Software Development (2018) 
Segundo o estudo empírico feito por Alqudah e Razali (2018), ao realizar um projeto de desenvolvimento de produto ou serviço, é raro o método do Scrum ou do Kanban se adequarem perfeitamente aos critérios do projeto em andamento. O Scrumban apoia os membros da equipe ágil a serem criativos no desenvolvimento de novos métodos para atender às suas necessidades. Não existem práticas específicas para o Scrumban, mas os membros da equipe ágil precisam entender quais práticas do Scrum e do Kanban agregam valor e escolhem as práticas apropriadas de acordo. O ScrumBan quando utilizado por alguém com experiência nos dois métodos, permite definir a duração dos sprints e limitar o Work-In-Process (WIP), que significa limitar a quantidade de tarefas em andamento.

\section{METODOLOGIA}

\subsection{Estudo de caso}

A metodologia de estudo de caso, segundo Voss, Tsikriktsis e Frohlich (2002) é indicado quando as variáveis investigadas ainda não são integralmente conhecidas, o fenômeno não é completamente compreendido e os resultados deste podem levar a diferentes percepções, gerando novas teorias relevantes. O presente estudo será realizado com casos múltiplos, que segundo Yin (2011) as provas resultantes de casos múltiplos são consideradas mais convincentes e o estudo global é visto como sendo mais robusto com relação ao estudo de caso único.

Para melhor conhecimento do contexto do trabalho, a startup $\mathrm{P}$, ainda nascente, possui 5 integrantes: o do comercial, CEO, que melhor representa o cliente perante o time todo, dono do produto (Product Owner - PO); o responsável pela gestão do projeto e auxilia no desenvolvimento do produto; e a equipe de desenvolvimento do produto efetivamente, que são 3 dos 5 envolvidos, 2 desenvolvedores (um cuida do backend / servidor, e o outro do aplicativo / frontend) e 1 designer (designer gráfico com ênfase em marketing). A equipe $\mathrm{P}$ trabalha à distância desde a sua criação. Já a empresa D é uma fábrica de software, com cerca de dois anos de existência, possui 14 integrantes divididos nas áreas de Tecnologia da Informação, marketing, produto e financeiro. A equipe $\mathrm{D}$ trabalha à distância desde o início da pandemia (em função do COVID 19), mas até o início de 2020 trabalhava presencialmente. É importante destacar que a empresa $\mathrm{D}$ foi utilizada neste trabalho como caso de sucesso, em função de usar a metodologia Scrumban e obter sucesso na sua aplicação. 
A pesquisa foi dividida em duas fases. Na primeira, o responsável pela empresa $\mathrm{P}$, que também é um dos membros do presente trabalho, coletou dados de duas empresas em entrevistas conjuntas e individuais. A empresa D está satisfeita com as suas entregas e vêm obtendo sucesso na aplicação da metodologia Scrumban, mas a empresa P está insatisfeita com suas entrega do desenvolvimento e buscaram por melhorias na gestão. O pesquisador usou dados da sua própria percepção e entrevistou os responsáveis diretos da gestão das duas empresas e da aplicação das mudanças, como CEO (Chief Executive Officer) e CTO (Chief Technology Officer). Estas entrevistas duraram cerca de 1 hora cada. Com isso, o responsável da empresa $\mathrm{P}$ e também pesquisador aplicou as principais ações usadas pela empresa $\mathrm{D}$ na empresa $\mathrm{P}$, de forma a melhorar o processo da desta última, que não estava com um bom desempenho no ambiente virtual. Já na segunda fase do trabalho, foi feita uma conjectura de melhorias na gestão da empresa P baseado na revisão teórica desse artigo e no bom funcionamento da gestão da empresa D.

\section{RESULTADOS E DISCUSSÃO}

\subsection{Análise do resultado do questionário e aplicação das mudanças}

Após a análise da primeira entrevista com as duas empresas, três fatores se destacaram com a startup P: falta de conhecimento de métodos de gestão além do Scrum, falha na comunicação e falta de controle do processo de gestão. Posterior a isso, os responsáveis pela implementação do estilo Scrumban de gestão realizam as mudanças em dois ciclos de melhorias testando algumas variações de combinações e chegaram no modelo ilustrado pela Figura 3, parecido com adotado pela empresa $\mathrm{D}$, de acordo com o CEO.

\begin{tabular}{|c|c|c|}
\hline \multirow{4}{*}{ Legenda } & Cor & Cenário da startup $\mathbf{P}$ \\
\cline { 2 - 3 } & Amarelo & Gestão antiga \\
\cline { 2 - 3 } & Verde & Gestão Nova \\
\cline { 2 - 3 } & Verde-amarelado & Gestão não alterada \\
\hline
\end{tabular}




\begin{tabular}{|c|c|c|c|}
\hline & Kanban & Scrum & Scrumban \\
\hline 1 & $\begin{array}{l}\text { Não há membros } \\
\text { predefinidos para } \\
\text { funções }\end{array}$ & $\begin{array}{l}\text { Funções predefinidas do } \\
\text { Scrummaster e membros da } \\
\text { equipe }\end{array}$ & $\begin{array}{l}\text { Funções predefinidas do } \\
\text { Scrummaster e membros da } \\
\text { equipe podem variar dentro do } \\
\text { tempo do projeto }\end{array}$ \\
\hline 2 & Entrega contínua & Sprints de tempo fixo & $\begin{array}{c}\text { Iterações baseadas em quadro } \\
\text { de tarefas }\end{array}$ \\
\hline 3 & $\begin{array}{l}\text { WIP limita a } \\
\text { quantidade de } \\
\text { trabalho }\end{array}$ & $\begin{array}{l}\text { Sprint limita a quantidade de } \\
\text { trabalho }\end{array}$ & $\begin{array}{l}\text { WIP limita a quantidade de } \\
\text { trabalho }\end{array}$ \\
\hline 4 & $\begin{array}{c}\text { Alterações podem } \\
\text { ser feitas a } \\
\text { qualquer } \\
\text { momento }\end{array}$ & $\begin{array}{c}\text { Nenhuma alteração permitida } \\
\text { durante os sprints }\end{array}$ & $\begin{array}{c}\text { Alterações permitidas no meio } \\
\text { do sprint }\end{array}$ \\
\hline 5 & $\begin{array}{l}\text { Planejamento e } \\
\text { documentação } \\
\text { anteriores } \\
\text { necessários }\end{array}$ & $\begin{array}{c}\text { Planejamento feito após cada } \\
\text { sprint }\end{array}$ & $\begin{array}{l}\text { Planejamento sob demanda, } \\
\text { também dentro de sprints }\end{array}$ \\
\hline 6 & $\begin{array}{l}\text { O quadro Kanban } \\
\text { é persistente }\end{array}$ & $\begin{array}{l}\text { O quadro scrum é redefinido } \\
\text { após cada sprint }\end{array}$ & $\begin{array}{l}\text { O quadro Scrumban é } \\
\text { persistente }\end{array}$ \\
\hline 7 & $\begin{array}{l}\text { O tamanho da } \\
\text { tarefa não é } \\
\text { limitado }\end{array}$ & $\begin{array}{c}\text { Tamanho da tarefa limitada a } \\
\text { uma impressão }\end{array}$ & $\begin{array}{c}\text { O tamanho da tarefa não é } \\
\text { limitado }\end{array}$ \\
\hline 8 & $\begin{array}{l}\text { Gerenciamento de } \\
\text { trabalho baseado } \\
\text { em puxar } \\
\end{array}$ & $\begin{array}{c}\text { Gerenciamento de trabalho } \\
\text { baseado em backlog da Sprint }\end{array}$ & $\begin{array}{l}\text { Gerenciamento de trabalho } \\
\text { baseado em puxar }\end{array}$ \\
\hline
\end{tabular}

FIGURA 3 - Situação pré e pós melhoria da gestão da Startup P. Fonte: Elaborado pelos autores

\subsubsection{Falta de conhecimento de métodos de gestão além do Scrum}

$\mathrm{O} C E O$, e $P O$, da startup $\mathrm{P}$ enfatizou na entrevista a falta de experiência em projetos na área do empreendedorismo digital, e que conta com a ajuda do $S M$, que está mais envolvido com a área, para direcionar o projeto à melhoria dos processos de gestão. O SM, ainda em formação em engenharia de produção, informou que não tinha conhecimentos mais aprofundados em outros métodos de gestão além do Scrum. A escolha de modificar o processo de gestão com base em outros métodos de gestão ágil veio de forma arbitrária por parte do $S M$ juntamente com o orientador do seu Trabalho de Conclusão de Curso, que possuía estrutura de trabalho semelhante de acordo com os entrevistados. 


\subsubsection{Falha na comunicação entre gestores e desenvolvedores}

Ao longo da entrevista com os gestores e desenvolvedores do projeto da startup $\mathrm{P}$, que adotam o Scrum como método de gestão, foi constatado que a equipe scrum (PO e o $S M$ ) trabalham de forma remota em função da distância entre os membros. Outro fato comentado foi que os envolvidos possuíam outros trabalhos, o que causava divergência de horários disponíveis entre eles nos dias úteis. Ao decorrer da entrevista percebeu-se que não havia um pleno acordo sobre o que estavam planejando para o produto. Também foi notada a ausência da comunicação diária (daily scrum) sobre as atividades executadas no dia devido a essa divergência de horários disponíveis. Logo, reuniões de 3 a 4 horas eram comuns aos domingos, dia no qual realizavam a sprint review e a sprint planning, de acordo com os entrevistados.

Estas percepções na falha da comunicação entre o time técnico e os gestores foram confirmadas pelas próprias palavras do CTO "o jeito que gerem os projetos na outra empresa que trabalho é o mesmo e só não temos os mesmos problemas que na startup $\mathrm{P}$ porque estamos sendo monitorados diariamente no próprio ambiente de produção". Já na empresa D, o CEO relata sobre o funcionamento da coleta de informações sobre a produtividade diária do time de desenvolvimento: "com a gente funciona muito bem relatar as atividades diárias através de aplicativo de mensagem", o que despertou o manifesto de interesse por parte da equipe da startup P como uma possível solução.

Outro ponto comentado por parte do time técnico da startup $\mathrm{P}$ foi a ausência de um software de gerenciamento mais visual dos backlogs a serem implementados no ciclo dos métodos, além de comentar sobre a recorrente não compreensão destes backlogs. Uma das gestoras da empresa D entrevistada contou sobre o auxílio do software de gerenciamento e criação de backlogs que utilizam e disse "ele nos ajuda a planejar, metrificar, e deixar visual as prioridades e o limite de backlogs em andamento".

\subsubsection{Falta de controle do processo de gestão}

A startup $\mathrm{P}$ julgou que a substituição de alguns princípios adotados do Scrum pelo do Kanban seja válida desde que o $S M$ se inteirasse dos novos processos e organizasse a mudança. Adaptaram a daily para ser realizada por mensagem de texto, assim como o case de sucesso da empresa D. Também, a empresa $\mathrm{P}$ adotou a estrutura visual de gestão dos quadros Kanban para as etapas do desenvolvimento (pré e durante), com limitação da carga de trabalho simultânea em desenvolvimento focando nas atividades prioritárias. Vale ressaltar que as iterações cíclicas do Scrum foram mantidas e combinadas à nova documentação e disposição visual das atividades nos quadros. 


\subsection{Discussão dos resultados}

A vontade e disposição da startup $\mathrm{P}$ se mostraram importantes para o resultado positivo percebido nas implementações das melhorias na gestão. Após a adaptação da daily sendo feita por mensagem de texto, foi constatado a melhoria da comunicação. Além disso, após a implementação da transição para os conceitos do Scrumban, foi percebida a melhora da visibilidade das atividades pré, durante e pós desenvolvimento por parte de todos da equipe devido a divisão dos backlogs em dois quadros: upstream e downstream.

Porém, foi notado que o software para a gestão adotado pela startup P limitava a visibilidade, tanto na quantidade de cartões de atividade, quanto em qualidade dos cartões em termos de priorização e integrações com os outros programas para o desenvolvimento. Logo, houve perda de potencial na melhoria por parte do software. Outro ponto de não melhoria constatado foi em relação ao entendimento dos backlogs por parte do time técnico, provavelmente devido à falta de inserções de imagens ou designs que exemplificassem o que deveria ser implementado, o que acha o CEO a empresa D.

\section{CONCLUSÃO}

Considerando o estudo feito nos dois empreendimentos digitais, constatou-se o destaque de três pontos marcantes: conhecimento da base teórica de variações da gestão ágil, comunicação e controle dos processos.

Os fatores de sucesso que contribuíram para a melhoria da comunicação foram: a adaptação da daily scrum (um dos princípios do Scrum) que a startup P conseguiu conciliar, além da melhoria na visibilidade das tarefas a serem executadas e em andamento. Entretanto, houveram fatores no quais reduziram o potencial de melhoria na comunicação e visualização do planejamento. Foi percebido ainda a não compreensão de tarefas por parte da equipe técnica da empresa $\mathrm{P}$, que se deu pela falha na explicação das tarefas sem um design representativo e pelo software "limitador" utilizado. Essa limitação foi responsável por dificultar a melhoria esperada e, devido à resistência da equipe em utilizar um software mais adequado, perderam potencial de alavancar o processo de gestão.

Sobretudo, é importante frisar que o conhecimento teórico que serviu de base para o artigo pode ser encontrado nas ferramentas de buscas acadêmicas de forma gratuita e hoje, muitos empreendedores tem o conhecimento sobre os métodos ágeis, em função da sua difusão. Além disso, foi notado uma rica troca de informações entre os empreendedores digitais, que se 
mostraram não receosos quando conversaram abertamente sobre os processos de gestões internas: o que mostra altruísmo entre essa classe de empresários.

\section{REFERÊNCIAS}

ABARCA, V. M. G.; PALOS-SANCHEZ, P. R.; RUS-ARIAS, E. Working in virtual teams: a systematic literature review and a bibliometric analysis. IEEE Access, v. 8, p. 168923-168940, 2020.

ALQUDAH, M.; RAZALI, R. An Empirical study of scrumban formation based on the selection of scrum and kanban practices. International Journal on advanced Science Engineering Information Technology, v.8, n.6, p.2315-2322, 2018.

AMBLER, S. Agile modeling: effective practices for extreme programming and the unified process. New York: JohnWiley, 2002.

CARVALHO, M. M.; RABECHINI JR., R. Fundamentos em gestão de projetos: construindo competências para gerenciar projetos. 3.ed. São Paulo: Atlas, 2011.

ANDERSON, D. J. Kanban. Sequim: Blue Hole, 2010.

FERRÃO, S. É. R.; CANEDO, E. D. A Study of the applicability of an agile methodology scrum allied to the Kanban method. In: IBERIAN, CONFERENCE ON INFORMATION SYSTEMS AND TECHNOLOGIES, 10 , 2015, Aveiro. Proceedings [...]. Piscataway: IEEE, 2015.

FOWLER, M. The New methodology. Wuhan University Journal of Natural Sciences, v. 6, n.1, p.12-24, 2001. GIONES, F.; BREM, A. Digital technology entrepreneurship: a definition and research agenda. Technology Innovation Management Review, v. 7, n. 5, p. 8, 2017.

KNIBERG, H.; SKARIN, M. Kanban and Scrum: making the most of both. Morrisville: Lulu, 2010.

SANJAY, P. P.; DR. JITESH R. N. Productivity Improvement of Software Development Process through Scrumban: A Practitioner's Approach. In: INTERNATIONAL CONFERENCE ON ADVANCES IN COMMUNICATION AND COMPUTING TECHNOLOGY, 2018, Ahmednagar. Proceedings [...]. Ahmednagar: IEEE, 2018 .

SCHWABER, K.; BEEDLE M. Agile software development with Scrum. Upper Saddle River: Prentice Hall, 2002.

SHALLOWAY, A. Demystifying kanban. Cutter IT Journal, v. 24, n. 3, p. 12, 2011.

SHEN, K. N.; LINDSAY, V.; XU, Y. C. Digital entrepreneurship. Information Systems Journal, v. 28, n. 6, p. 1125-1128, nov. 2018.

SOUZA, M. L. P.; MELO FILHO, L. D. R.; OLIVEIRA, C. G.; ANICETO, M. D.; SILVEIRA, C. Á. Aplicação conjunta de métodos no desenvolvimento de startups: descrição e análise crítica. In: CONGRESSO

BRASILEIRO DE INOVAÇÃO E GESTÃO DE DESENVOLVIMENTO DO PRODUTO, 11., São Paulo.

Anais [...]. São Paulo: Blucher, 2017. v. 3, n. 12, p. 725-734, 2017.

SUTHERLAND, J.; SCHWABER, K. The Scrum guide. The Definitive guide to scrum: the rules of the game. [S.l.]: Scrum. org, 2013.

TARNE, R. Taking off the agile training wheels, advance agile project management using Kanban. In: PMI GLOBAL CONGRESS, 2011, Dallas. Proceedings [...]. Newtown Square: Project Management Institute, 2011.

VOSS, C; TSIKRIKTSIS, N; FROHLICH, M. Case research in operations management. International Journal of Operations \& Production Management, v.22, n.2, p.195-219, 2002.

YIN, R. K. Qualitative research from start to finish. New York: Guilford, 2011.

ZAHEER, H.; BREYER, Y.; DUMAY, J. Digital entrepreneurship: an interdisciplinary structured literature review and research agenda. Technological Forecasting and Social Change, v. 148, Nov. 2019. DOI: https://doi.org/10.1016/j.techfore.2019.119735. 